\title{
Functional kidney progenitors can be isolated from preterm urine
}

Urine from preterm neonates collected before the completion of nephrogenesis - offers a novel source of kidney progenitor cells, with the ability to differentiate into mature renal cells, according to data published in the Journal of the American Society of Nephrology.

Previous studies have shown ...nKSPC-podo cells were highly active and able to endocytose albumin as well as demonstrating calcium influx... amniotic fluid and adult urine progenitor cells to act as controls.

Genetic characterisation showed expression of the kidney progenitor genes SIX2, CITED2 and VIM in cells isolated from amniotic fluid and neonatal kidney stem/progenitor cells (nKSPCs) - SIX2 was specifically expressed in urine from neonates born before 34 weeks, and was not present in adult cells. Single cell RT-PCR showed expression of several renal progenitor markers in addition to SIX2 (expressed in $17.5 \%$ of cells): FOXD1 (15\% ), OSR1 (10\%), and PAX2 (20\%). 7.5\% of nKSPCs were SIX2+/FOXD1+.

The team then went on to investigate the function of the isolated nKSPCs. First, they showed that co-culture of nKSPCs and conditionally immortalized proximal tubule cells with cisplatin protected against cisplatin-induced apoptosis. They also showed differentiation of KSPCs into podocytes, with higher expression of podocyte-specific genes in those derived from nKSPCs (nKSPC-podo) compared with those from other sources. Furthermore, nKSPC-podo cells were highly active and able to endocytose albumin as well as demonstrating calcium influx via an active TrpC6 channel (a podocyte-specific transient receptor potential cation channel).

As nKSPCs can be noninvasively collected, expanded, and stored for future use, they could have potential for regenerative repair of the kidney, especially as preterm infants are at risk of developing renal insufficiency later in life.

Annette Fenner

ORIGINAL ARTICLE Arcolino, F. O. et al. Urine of preterm meonates as a novel source of kidney progenitor cells.J. Am. Soc. Nephrol. http://dx.doi. org/10.1681/ASN.2015060664 (2016) 